\title{
Comparison of Two Porcine Collagen Membranes Combined with rhBMP-2 and rhBMP-9 on Osteoblast Behavior In Vitro
}

\author{
Masako Fujioka-Kobayashi, DDS, $\mathrm{PhD}^{1}$ /Benoit Schaler, MD, DDS ${ }^{2} /$ Yoshinori Shirakata, DDS, $\mathrm{PhD}^{3}$ / \\ Toshiaki Nakamura, DDS, $\mathrm{PhD}^{3} /$ Kazuyuki Noguchi, DDS, $\mathrm{PhD}^{4} /$ \\ Yufeng Zhang, DDS, MD, PhD $5 /$ Richard J. Miron, DDS, $\mathrm{PhD}^{6}$
}

\begin{abstract}
Purpose: To investigate the bone-inducing properties of two types of collagen membranes in combination with recombinant human bone morphogenetic protein (rhBMP)-2 and rhBMP-9 on osteoblast behavior. Materials and Methods: Porcine pericardium collagen membranes (PPCM) and porcine dermis-derived collagen membranes (PDCM) were coated with either rhBMP-2 or rhBMP-9. The adsorption and release abilities were first investigated via enzyme-linked immunosorbent assay up to 10 days. Moreover, murine bone stromal ST2 cell adhesion, proliferation, and osteoblast differentiation were assessed by MTS assay; real-time polymerase chain reaction for genes encoding runt-related transcription factor 2 (Runx2); alkaline phosphatase (ALP); and osteocalcin, ALP assay, and alizarin red staining. Results: Both rhBMP-2 and rhBMP-9 adsorbed to collagen membranes and were gradually released over time up to 10 days. PPCM showed significantly less cell attachment, whereas PDCM demonstrated comparable cell attachment with the control tissue culture plastic at 8 hours. While both rhBMPs were shown not to affect cell proliferation, collagen membranes combined with rhBMP-9 significantly increased ALP activity at 7 days and ALP mRNA levels at either 3 or 14 days compared with the control tissue culture plastic. Furthermore, rhBMP-9 increased osteocalcin mRNA levels and alizarin red staining at 14 days compared with the control tissue culture plastic. Conclusion: The results from this study suggest that both porcine-derived collagen membranes combined with rhBMP-9 accelerated the osteopromotive potential of ST2 cells. Interestingly, rhBMP-9 demonstrated additional osteogenic differentiation compared with rhBMP-2 and may serve as a suitable growth factor for future clinical use. INT J ORAL MaXILLOFAC IMPLANTS 2017;32:e221-e230. doi: 10.11607/jomi.5652
\end{abstract}

Keywords: bone regeneration, BMP-2, BMP-9, collagen membrane, guided bone regeneration, porcine collagen membrane

\footnotetext{
${ }^{1}$ Assistant Professor, Department of Cranio-Maxillofacial Surgery, Bern University Hospital, Inselspital, Bern, Switzerland; Department of Oral Surgery, Institute of Biomedical Sciences, Tokushima University Graduate School, Tokushima, Japan.

${ }^{2}$ Associate Professor, Department of Cranio-Maxillofacial Surgery, Bern University Hospital, Inselspital, Bern, Switzerland.

${ }^{3}$ Associate Professor, Department of Periodontology, Kagoshima University Graduate School of Medical and Dental Sciences, Kagoshima, Japan.

4Professor, Department of Periodontology, Kagoshima University Graduate School of Medical and Dental Sciences, Kagoshima, Japan.

${ }^{5}$ Professor, Department of Oral Implantology, School of Stomatology, Wuhan University, Wuhan, China.

${ }^{6}$ Professor, Department of Periodontology, College of Dental Medicine, Nova Southeastern University, Fort Lauderdale, Florida, USA.
}

Correspondence to: Dr Richard Miron, Department of Periodontology, College of Dental Medicine, Nova Southeastern University, 3301 College Avenue, Fort Lauderdale, FL 33314, USA. Email: rmiron@nova.edu

@2017 by Quintessence Publishing Co Inc.
Collagen membranes are widely used for bone and connective tissue augmentation techniques such as guided tissue regeneration/guided bone regeneration (GBR) due to their biocompatibility and wound healing capabilities. ${ }^{1-4}$ Collagen membranes promote the rapid ingrowth and repopulation of various types of cells from the surrounding tissues by creating a space-making ability later used for tissue repopulation. ${ }^{1,5,6}$ Commonly utilized membranes include porcine-derived collagen membranes, which are approved for soft tissue, connective tissue, and bone tissue regeneration, and represent necessary biomaterials commonly utilized in periodontal and implant surgery. 7,8 Various types of collagen membranes are available, including porcine pericardium collagen membrane (PPCM) and porcine dermis-derived collagen membrane (PDCM). These collagen membranes are constructed of native collagen type I/III without artificial crosslinking and provide ideal properties for vascular formation with the ability to degrade over 
time. ${ }^{9,10}$ Nevertheless, the regenerative potential of collagen membranes is limited to their osteoconductive surfaces with no osteoinductive potential. ${ }^{11}$

For these reasons, the use of collagen membranes is often combined with recombinant human bone morphogenetic proteins (rhBMPs) to increase their osteoinductive and osteopromotive potential. ${ }^{11}$ They are considered ideal carriers for growth factor delivery able to release recombinant proteins over time as well as during their gradual degradation. ${ }^{12}$ rhBMP-2 has been the most commonly used growth factor for bone regenerative procedures in dentistry during GBR techniques due to its potent osteoinductive potential. ${ }^{13,14}$ Furthermore, in many studies, collagen products have been shown to successfully act as efficient carriers for rhBMP-2 delivery. ${ }^{15-19}$ Despite the widespread use of rhBMP-2, many investigators may be surprised to learn that previous adenovirus experiments (gene therapy) have actually shown that BMP-9 induces greater osteogenic potential compared with BMP-2. ${ }^{20-23}$ While these previous studies utilized adenovirus transfection experiments still not approved by the U.S. Food and Drug Administration, ${ }^{20-23}$ the present group of authors recently reported that rhBMP-9 also strongly accelerated osteoblast differentiation compared with rhBMP-2.24,25

Therefore, the aim of the present study was to determine which type of collagen membrane (PPCM or PDCM) has a better influence on the adsorption/release of rhBMPs, as well as its effect on cell behavior.

\section{MATERIALS AND METHODS}

\section{Reagents and Cell Line}

Recombinant human BMP-2 and BMP-9 were purchased from R\&D Systems. The native PPCM utilized (Jason membrane) and the porcine dermis collagen membrane (PDCM) utilized (mucoderm) were both kindly provided by Botiss. Both products are composed of mainly collagen type I, and to a lower degree, collagen type III. For all in vitro experiments, the following four groups for each collagen membrane were examined: (1) tissue culture plastic (control), (2) collagen membrane only, (3) collagen membrane + BMP-2 $(100 \mathrm{ng} / \mathrm{mL})$, and (4) collagen membrane + BMP-9 (100 $\mathrm{ng} / \mathrm{mL}$ ). Undifferentiated mouse bone stromal cell-line ST2 was obtained from RIKEN Cell Bank. Cells were cultured in a humidified atmosphere at $37^{\circ} \mathrm{C}$ in growth medium consisting of Dulbecco's modified Eagle's medium (DMEM; Gibco, Life Technologies), 10\% fetal bovine serum (FBS; Gibco), and antibiotics (Gibco). Each collagen membrane was placed at the bottom of 24well plates and precoated with rhBMP-2 or rhBMP-9 in DMEM for 5 minutes prior to cell seeding. Then, cells were seeded at a density of 10,000 cells in 24-well plates for cell adhesion and proliferation experiments and 50,000 cells per well in 24-well plates for real-time polymerase chain reaction (PCR), alkaline phosphatase (ALP) assay, and alizarin red experiments. For experiments lasting longer than 5 days, the medium was replaced twice weekly.

\section{Scanning Electron Microscopy Images}

Collagen membrane samples were sputter-coated using an ion coater device with $10 \mathrm{~nm}$ of gold and analyzed microscopically using a scanning electron microscope as previously described. ${ }^{26}$

\section{BMP-2 and BMP-9 Adsorption and Release Kinetics Quantification with ELISA}

To determine the quantity of rhBMP-2 and rhBMP-9 adsorption to PPCM or PDCM, enzyme-linked immunosorbent assay (ELISA) quantification assay was utilized. Briefly, after the coating period incubation of $100 \mathrm{ng} /$ $\mathrm{mL}$ of rhBMP-2 or rhBMP-9 onto PPCM and PDCM at $37^{\circ} \mathrm{C}$ in a shaking incubator, the remaining phosphatebuffered saline solution, containing unattached protein, was collected and quantified by an ELISA Duoset kit for BMP-2 (DY355; range $=46.90$ to $3,000 \mathrm{pg} / \mathrm{mL}$, R\&D Systems) and BMP-9 (DY3209; range $=15.60$ to $1,000 \mathrm{pg} / \mathrm{mL}$, R\&D Systems). Subtraction of the total coated protein from the amount of unadsorbed protein was used to determine the amount of adsorbed material to the surface of PPCM and PDCM as previously described. ${ }^{27}$ Furthermore, to determine the quantity of rhBMP-2/rhBMP-9 protein being released from PPCM and PDCM over time, coated grafts were soaked in 1 $\mathrm{mL}$ of phosphate-buffered saline, and samples were collected at various time points including 15 minutes, 1 hour, 8 hours, 24 hours, 3 days, and 10 days. All samples were quantified in duplicate, and three independent experiments were performed.

\section{Adhesion and Proliferation Assay}

Cells were quantified using an MTS assay (Promega) using an ELx808 Absorbance Reader (BIO-TEK) at 8 hours for cell adhesion and at 1, 3, and 5 days for cell proliferation after cell seeding as previously described. ${ }^{28}$

\section{Real-Time PCR Analysis for Osteoblast Differentiation Markers}

Total RNA was isolated using High Pure RNA Isolation Kit (Roche) at 3 and 14 days for osteoblast differentiation markers. Primers of runt-related transcription factor 2 (Runx2), ALP, osteocalcin, and glyceraldehyde 3-phosphate dehydrogenase (GAPDH) were fabricated with primer sequences according to Table 1. Real-time PCR was performed using Roche FastStart Universal SYBR Green Master and quantified on an Applied 
Biosystems 7500 Real-Time PCR Machine (Biosystems, Life Technologies). A Nanodrop 2000c (Thermo) was used to quantify total RNA levels. The $\Delta \Delta \mathrm{Ct}$ method was used to calculate gene expression levels normalized to GAPDH values and calibrated to control samples.

\section{ALP Stain Assay}

At 7 days, ALP activity was monitored using Leukocyte alkaline phosphatase kit (procedure No. 86 , Sigma) as previously described. ${ }^{25}$ ST2 cells were fixed by immersion in a citrate-acetone-formaldehyde fixative solution for $5 \mathrm{~min}$ utes. Alkaline dye mixture was prepared by mixing $1 \mathrm{~mL}$ of sodium nitrite solution and $1 \mathrm{~mL}$ of fast red violet alkaline solution dissolved in $45 \mathrm{~mL}$ of deionized water and 1 $\mathrm{mL}$ of Naphtol AS-BI alkaline solution. Surfaces were then placed in alkaline dye mixture solution for 20 minutes protected from light followed by rinsing in deionized water. All images were captured on a Wild Heerbrugg M400 ZOOM Makroskop (Wild Heerbrugg) at the same magnification and light intensity and imported into ImageJ software (National Institutes of Health). Thresholding was used to generate percent stained values for each field of view.

\section{Mineralization Assay}

ST2 cells were seeded in 24-well plates at a density of 50,000 cells per well on either membrane in osteogenic differentiation medium, which consisted of DMEM supplemented with $10 \% \mathrm{FBS}, 1 \%$ antibiotics, $50 \mu \mathrm{g} / \mathrm{mL}$ ascorbic acid (Sigma), and $10 \mathrm{mM} \beta$-glycerophosphate (Sigma) to promote osteoblast differentiation as previously described. ${ }^{29}$ At 14 days after ST2 cell seeding, cells were fixed in $96 \%$ ethanol for 15 minutes and stained with $0.2 \%$ alizarin red solution (Alizarin Red S, Sigma) in deionized water ( $\mathrm{pH}$ 6.4) at room temperature for 1 hour as previously described. ${ }^{25,29}$ All images were captured and the percentage of staining was evaluated in the same manner as the ALP assay.

\section{Statistical Analysis}

All experiments were performed in triplicate with three independent experiments for each condition. Data were analyzed for statistical significance using one-way analysis of variance with the Tukey test $\left({ }^{*} P<\right.$ .05 was considered significant) by GraphPad Prism 6.0 software (GraphPad Software).

\section{RESULTS}

\section{Surface Characteristics of Collagen Membranes and BMP Adsorption and Release Kinetics}

In a first experiment, the surface morphology of PPCM and PDCM was investigated via scanning electron microscopy (Fig 1). The surface of PPCM demonstrated a three-dimensional (3D) porous structure with

\begin{tabular}{lc} 
Table 1 & $\begin{array}{l}\text { PCR Primers for Genes Encoding } \\
\text { Runx2, ALP, OCN, and GAPDH }\end{array}$ \\
\hline Gene & \multicolumn{1}{c}{ Primer sequence } \\
mRunx2 F & agggactatggcgtcaaaca \\
\hline mRunx2 R & ggctcacgtcgctcatctt \\
\hline mALP F & ggacaggacacacacacaca \\
\hline mALP R & caaacaggagagccacttca \\
\hline mOCN F & cagacaccatgaggaccatc \\
\hline mOCN R & ggactgaggctctgtgaggt \\
\hline mGAPDH F & aggtcggtgtgaacggatttg \\
\hline mGAPDH R & tgtagaccatgtagttgaggtca \\
\hline
\end{tabular}

Runx2 = runt-related transcription factor 2; ALP = alkaline phosphatase; $\mathrm{OCN}=$ osteocalcin; GAPDH = glyceraldehyde 3-phosphate dehydrogenase.

numerous collagen fibers (Figs $1 \mathrm{a}$ and $1 \mathrm{c}$ ). PDCM, on the other hand, demonstrated a more densely structured outer surface with few pores (Figs $1 \mathrm{~b}$ and $1 \mathrm{~d}$ ). Thereafter, the potential of PPCM and PDCM to adsorb and release BMP-2 and BMP-9 was investigated by ELISA (Fig 2). The total amount of adsorbed BMP-2 and BMP-9 to collagen membrane after 5 minutes revealed a nearly $90 \%$ adsorption for both collagen membranes, and after a 10-day period, approximately $50 \%$ of the initial BMP-2 and BMP-9 loaded onto collagen membranes was released (Figs 2a and 2b). Interestingly, BMP-2 showed significantly more adsorption and retention over time up to 10 days on PPCM compared with PDCM (Fig 2a). On the contrary, BMP-9 demonstrated no significant difference in its adsorption between PPCM and PDCM but higher retention on PDCM from 1 hour up to 10 days compared with PPCM (Fig 2b).

\section{Effects of rhBMP-2 and rhBMP-9 Loaded Collagen Membranes on ST2 Cell Adhesion and Proliferation}

Thereafter, the effects of rhBMP- 2 and rhBMP-9 loaded on collagen membranes were investigated on ST2 cell adhesion at 8 hours and cell proliferation at 1, 3, and 5 days postseeding (Fig 3). It was first observed that PPCM significantly decreased ST2 cell attachment compared with the control at 8 hours after cell seeding (Fig 3a). Interestingly, cell adhesion increased on collagen membrane + BMP-2 samples compared with PPCM alone or PPCM + BMP-9 (Fig 3a). While the cell number was decreased on PPCM, PDCM displayed no significant changes between all groups, and thereby, better cell attachment was observed (Fig 3b). Moreover, PPCM and PDCM were tested for their abilities to induce cell proliferation (Figs $3 \mathrm{c}$ and $3 \mathrm{~d}$ ). While both PPCM and PDCM significantly inhibited cell growth compared with the control, it was once again observed 

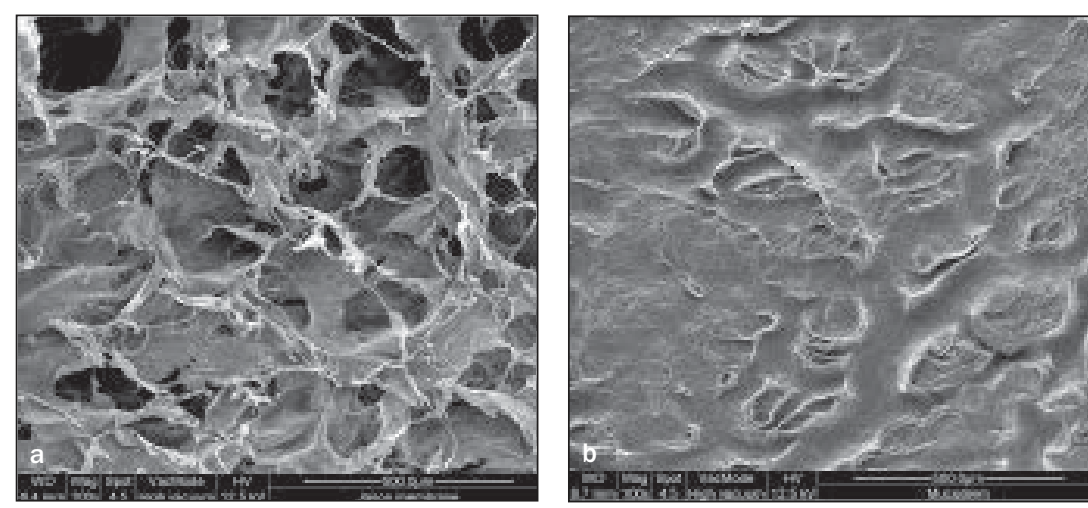

Fig 1 Scanning electron microscopy images of $(a, c)$ PPCM and $(b, d)$ PDCM at low $(100 \times)$ and high $(400 \times)$ magnification. PPCM membranes showed a lace-like collagen fibril three-dimensional surface morphology, whereas PDCM membranes showed a more flattened surface.
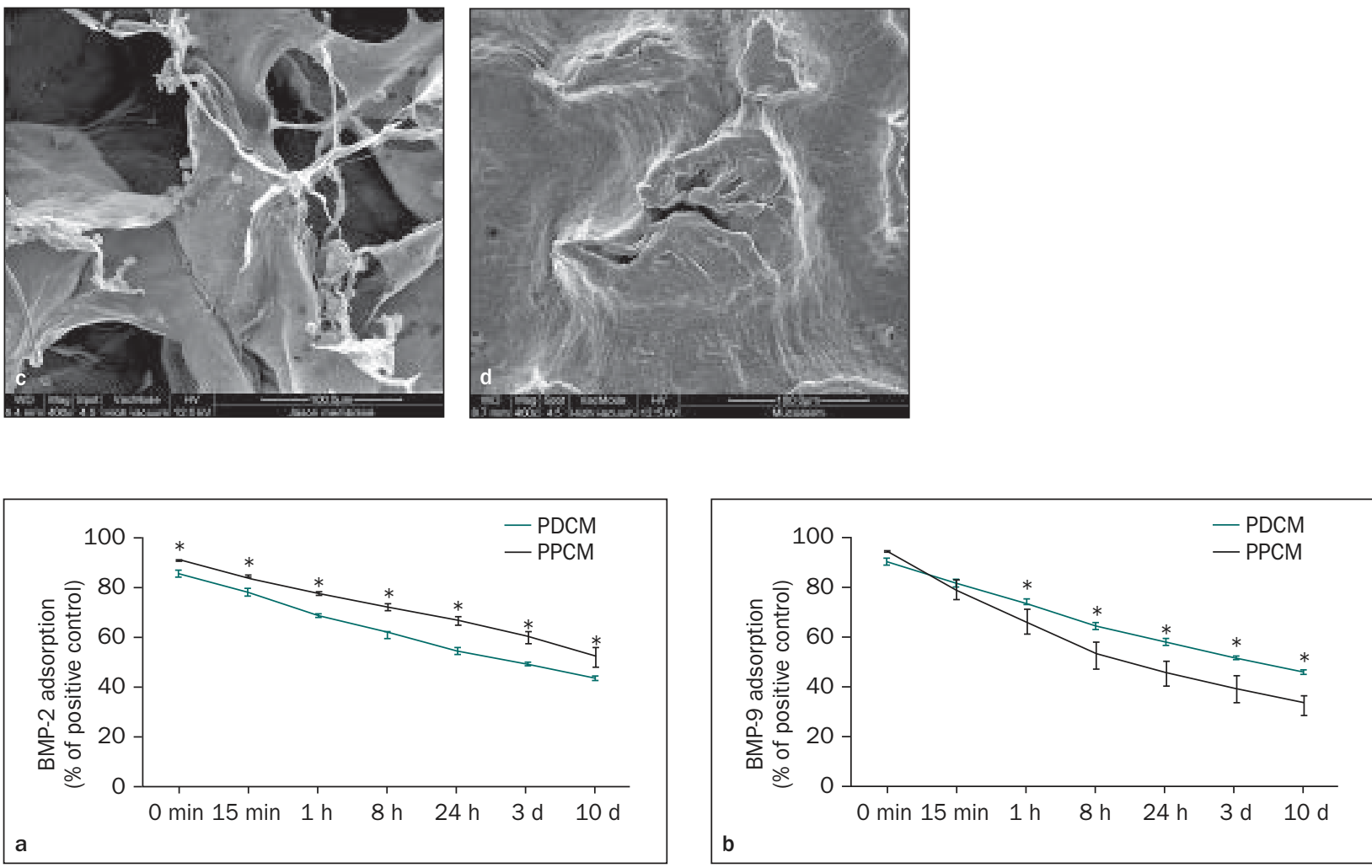

Fig 2 (a) rhBMP-2 and (b) rhBMP-9 adsorption potential when loaded onto PPCM and PDCM at 15 minutes, 60 minutes, 8 hours, 24 hours, 3 days, and 10 days (*denotes significantly higher than the other group, $P<.05$ ).

that cell numbers were higher on PDCM compared with PPCM (Figs $3 c$ and $3 d$ ).

\section{Effects of rhBMP-2 and rhBMP-9 Loaded onto Collagen Membranes on Osteoblastic Differentiation}

Thereafter, the osteogenic differentiation potentials of rhBMP-2 and rhBMP-9 precoated on PPCM and PDCM were investigated by ALP activity (Fig 4), realtime PCR (Fig 5), and alizarin red staining (Fig 6). It was first observed that both collagen membranes alone did not affect ALP activity compared with the control (Fig 4). The additional combination of rhBMP-2 and rhBMP-9 induced higher levels of ALP activity compared with collagen membranes alone or the control (Figs $4 \mathrm{~g}$ and $4 \mathrm{~h}$ ). Interestingly, while rhBMP-9 significantly upregulated ALP activity on both collagen membranes, rhBMP-2 was only able to significantly upregulate ALP activity on PDCM membranes (Figs $4 \mathrm{~g}$ and $4 \mathrm{~h}$ ).

While all PDCM groups nonsignificantly decreased Runx2 mRNA levels at 3 days, no difference was observed at 14 days compared with the control (Figs $5 a$ and 5b). Interestingly, PPCM + BMP-9 samples at 14 days and PDCM + BMP-9 at 3 days upregulated ALP mRNA levels up to fourfold compared with the control (Figs $5 c$ and $5 \mathrm{~d}$ ). In a similar manner, collagen membranes + BMP-9 samples significantly increased 

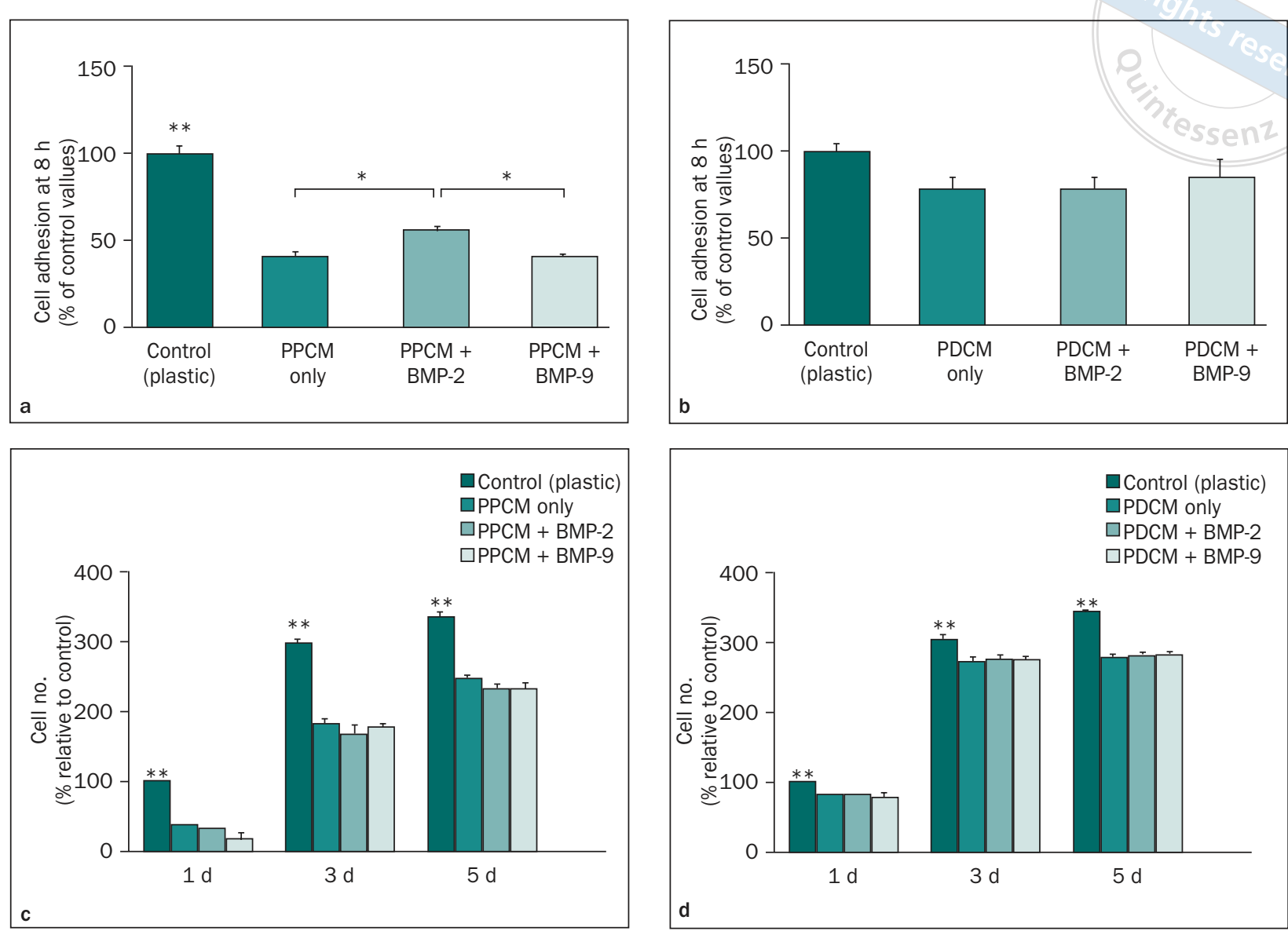

Fig 3 Cell adhesion and proliferation assay of ST2 cells seeded on collagen membrane combined with rhBMP-2 and rhBMP-9. (a, b) ST2 cell attachment on (a) PPCM or (b) PDCM with (1) control, tissue culture plastic; (2) collagen membrane only; (3) BMP-2 (100 $\mathrm{ng} / \mathrm{mL})$; (4) BMP-9 (100 ng/mL) at 8 hours after cell seeding. (c, d) Cell proliferation on ST2 cell attachment on (c) PPCM or (d) PDCM with (1) control, tissue culture plastic; (2) collagen membrane only; (3) BMP-9 (100 ng/mL); (4) BMP-9 (100 ng/mL) at 1, 3, and 5 days after cell seeding. (*denotes significant difference, $P<.05 ; * *$ denotes significantly higher than all other modalities, $P<.05)$.

osteocalcin mRNA levels at 14 days postseeding compared with the control, whereas no difference was observed with rhBMP-2 (Figs $5 e$ and $5 f$ ).

Lastly, alizarin red staining was performed to assess mineralization potential of rhBMP-2 and rhBMP-9 when coated onto both collagen membranes (Fig 6). It was found at 14 days after cell seeding that on PPCM membranes, rhBMP-9 induced significantly higher alizarin red staining compared with the control and PPCM membranes alone, whereas PDCM membranes with either rhBMP-2 or rhBMP-9 induced significantly higher alizarin red staining compared with the control or PDCM membranes alone (Figs $6 \mathrm{e}$ to $6 \mathrm{~h}$ and $6 \mathrm{j})$.

\section{DISCUSSION}

The aim of the present study was to assess the boneinducing properties of two porcine-derived collagen membranes commonly utilized in regenerative dentistry when loaded with rhBMP-2 and rhBMP-9. In general, bone augmentation procedures have been characterized as derived from four principal methods including: (1) osteoinduction, which utilizes appropriate growth factors; (2) osteoconduction, which is the ability for the scaffold to conduce new bone formation; (3) distraction osteogenesis, which generates new bone by progressive stretching of divided bone segments; and (4) guided tissue 


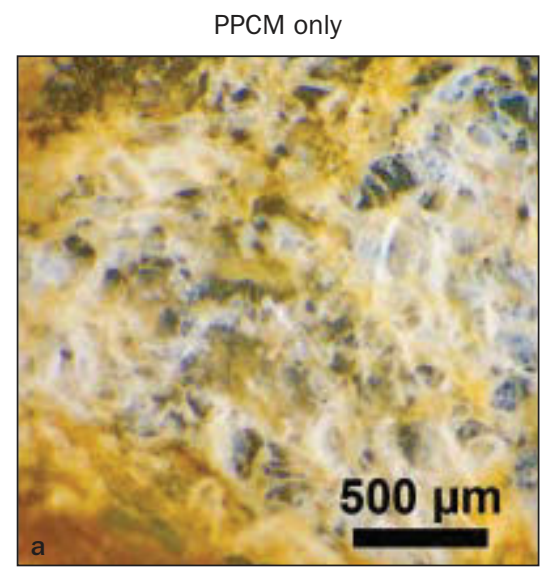

PDCM only

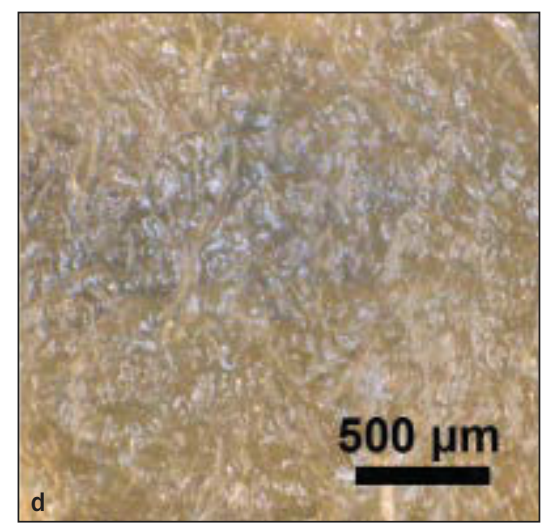

PPCM + BMP-2

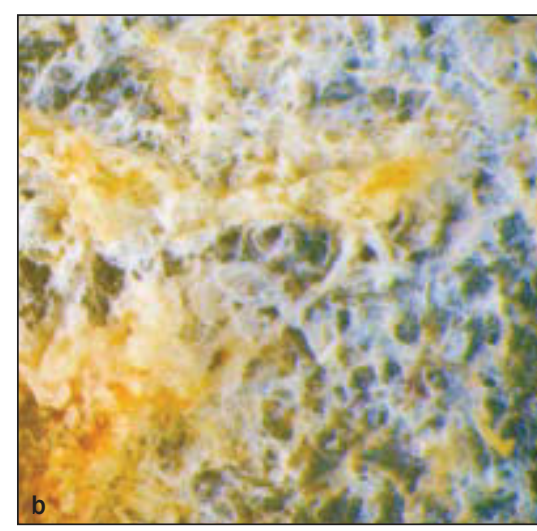

PDCM + BMP-2

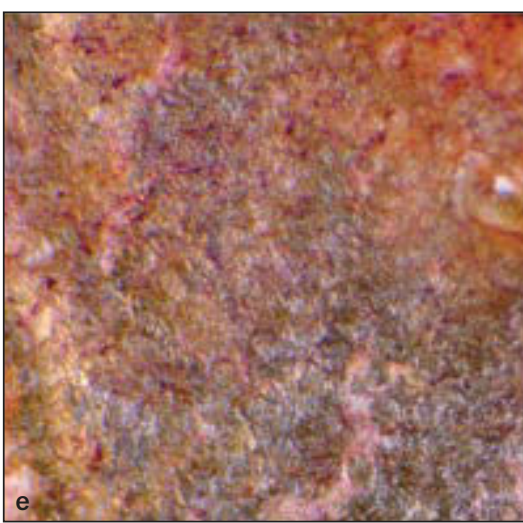

PPCM + BMP-9

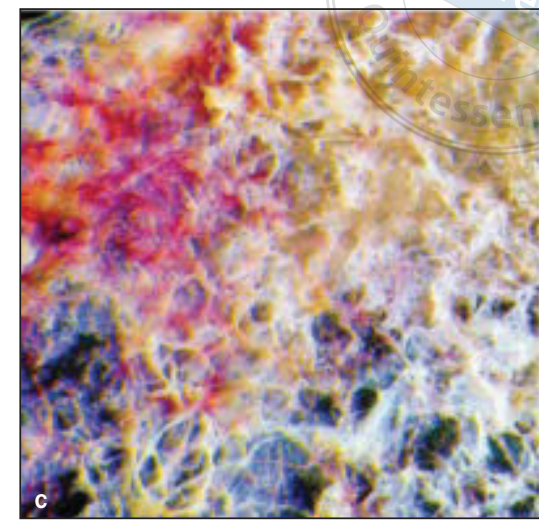

PDCM + BMP-9

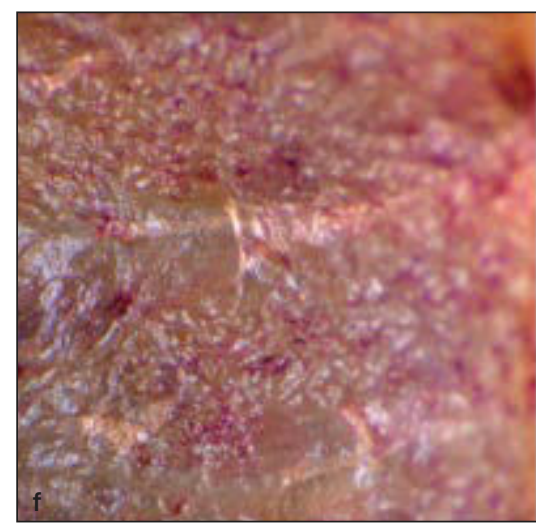

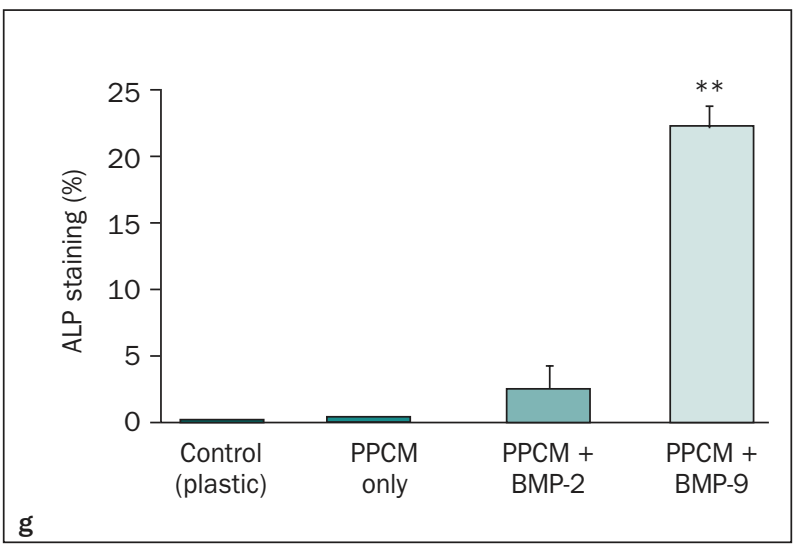

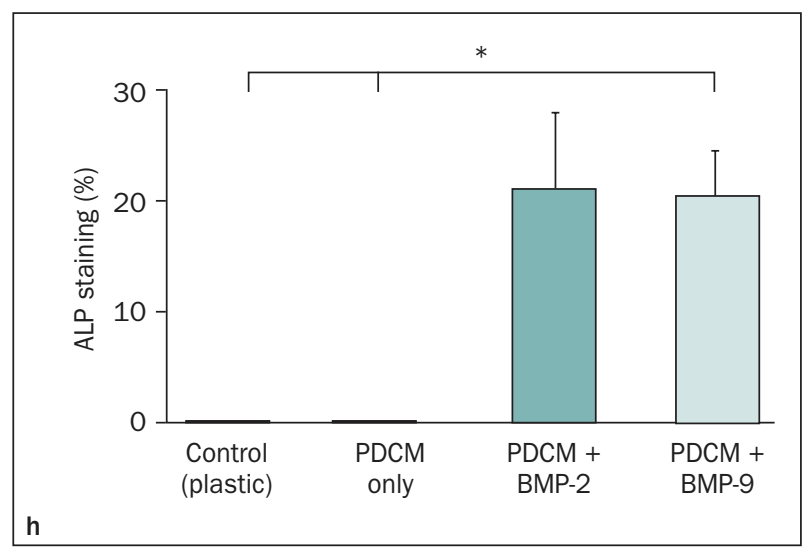

Fig 4 (a to $f$ ) Image views and ( $g, h$ ) quantified data of alkaline phosphatase (ALP) staining of ST2 cells on (a to $c$, g) PPCM or (d to $f$, h) PDCM with (1) control, tissue culture plastic; (2) collagen membrane only; (3) BMP-2 (100 ng/mL); (4) BMP-9 (100 ng/mL) at 7 days after cell seeding. (*denotes significant difference, $P<.05 ; * *$ denotes significantly higher than all other treatment modalities, $P<.05)$.

regeneration/GBR, which allows space maintenance by barrier membranes to be later occupied with bone. ${ }^{30}$ The present study investigated the osteogenic possibilities of rhBMP-2 and rhBMP-9 as osteoinductive factors, and two kinds of porcine native collagen membranes as GBR membranes, thereby directly assessing the bone augmentation principles (1), (2), and (4).
The first set of experiments investigating the surface morphologic features of both PPCM and PDCM demonstrated 3D constructs with numerous collagen fibers expecting for cell ingrowth, namely, in the PPCM (Fig 1). Interestingly, PDCM showed a denser structure compared with PPCM, and these differences are discussed later as potential reasons for the observed cellular differences. Extracellular collagenous matrix acts 

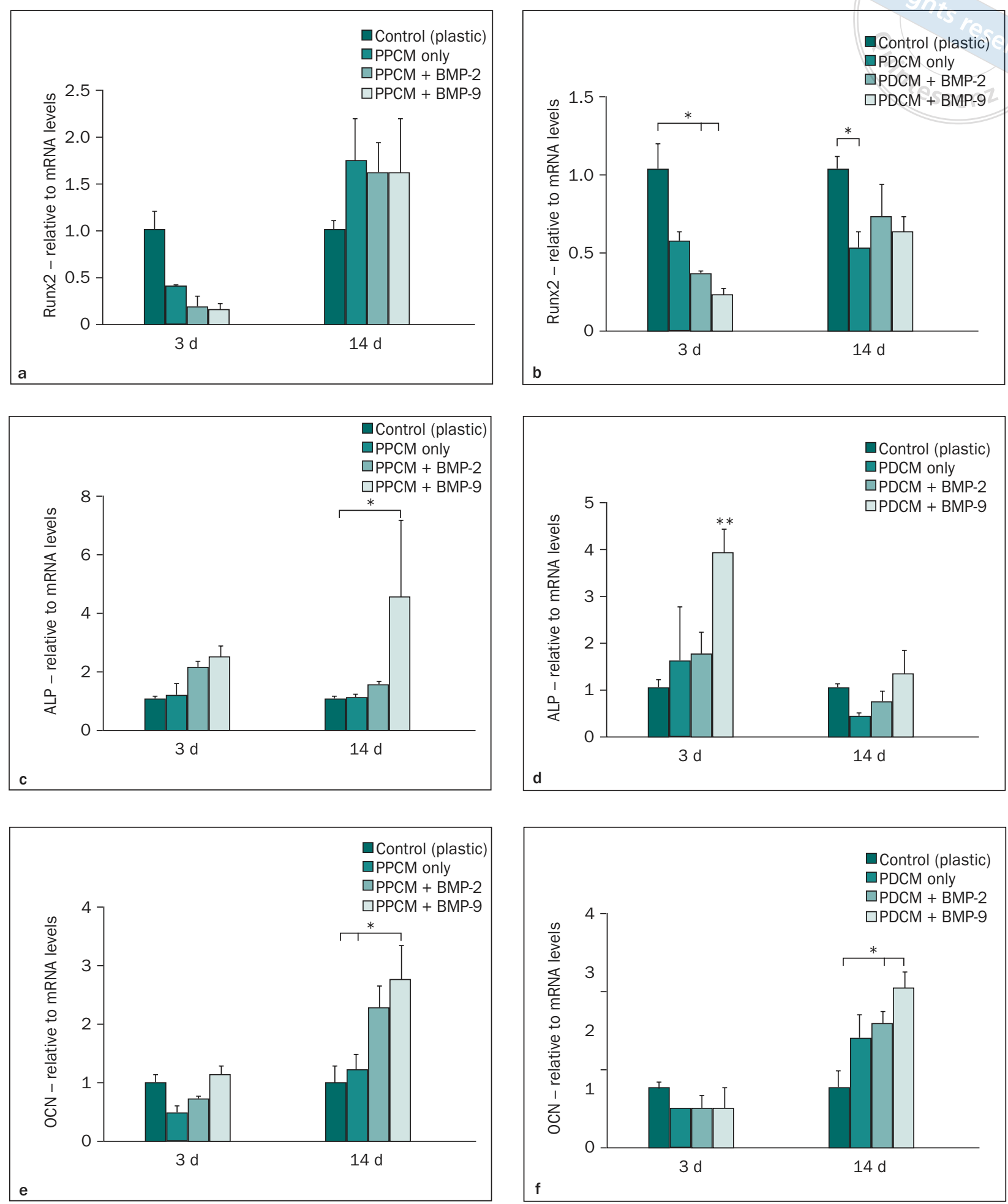

Fig 5 Real-time PCR of ST2 cells seeded on (a, c, e) PPCM or (b, $d, f)$ PDCM with (1) control, tissue culture plastic; (2) collagen membrane only; (3) BMP-2 (100 ng/mL); (4) BMP-9 (100 ng/mL) for genes encoding (a, b) Runx2, (c, d) ALP, (e, f) osteocalcin (OCN) at 3 and 14 days after seeding (*denotes significant difference, $P<.05 ; * *$ denotes significantly higher than all other treatment modalities, $P<.05)$. 


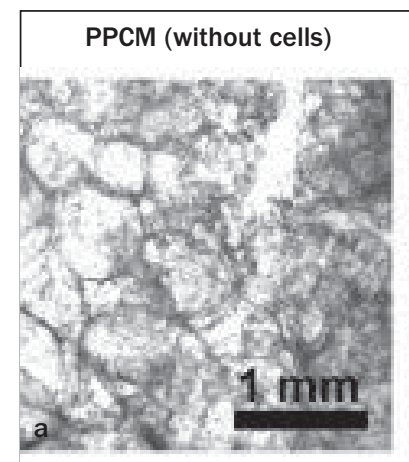

PPCM + BMP-2

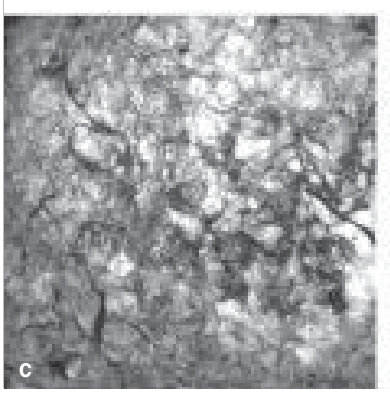

PPCM (with cells)

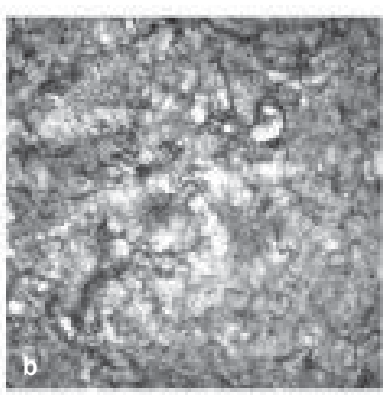

PPCM + BMP-9

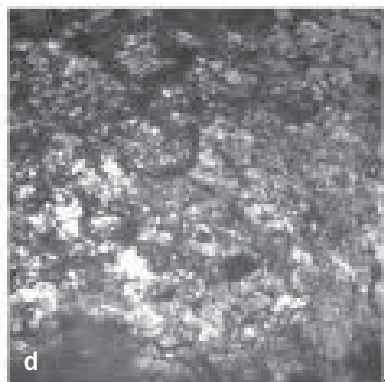

PDCM (without cells)

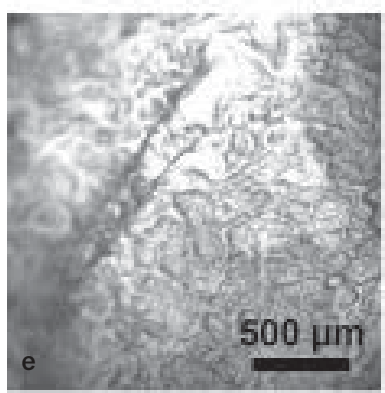

PDCM + BMP-2

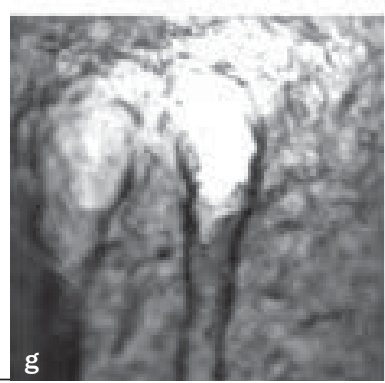

PDCM (with cells)

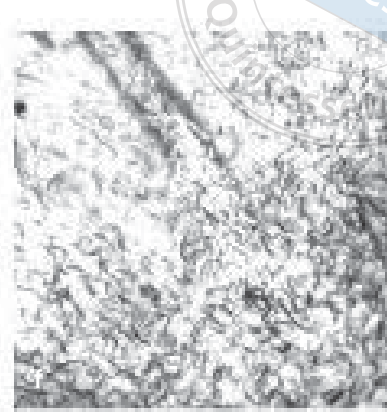

PDCM + BMP-9

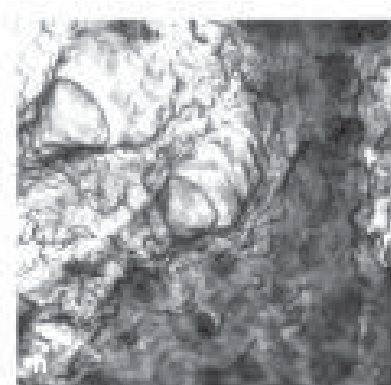

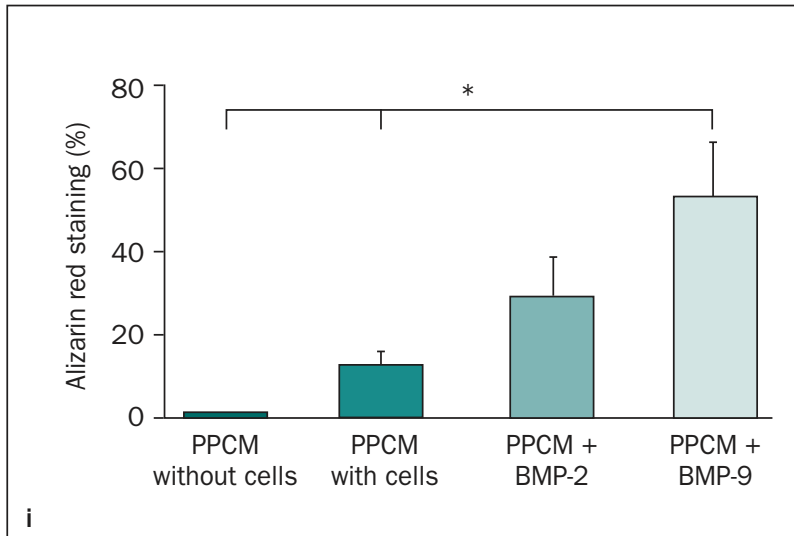

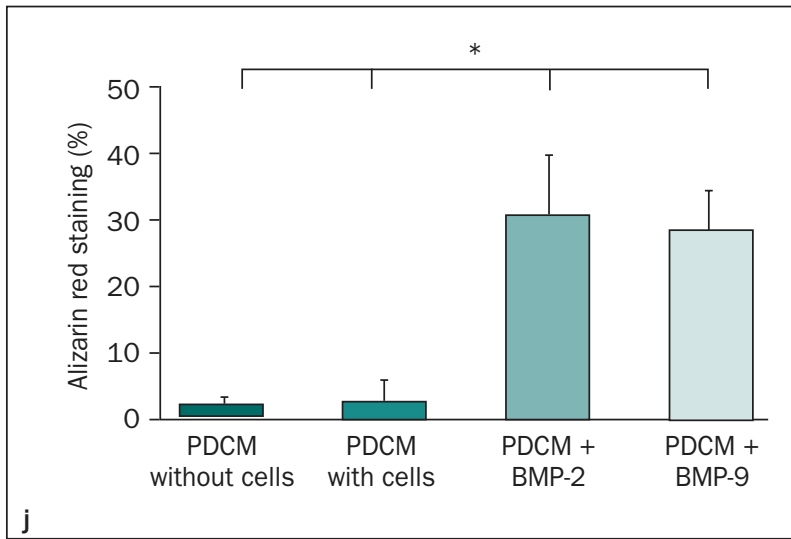

Fig 6 Alizarin red staining of ST2 cells on (a to e, i) PPCM or (e to $h$, j) PDCM with (1) control, tissue culture plastic; (2) collagen mem brane only; (3) BMP-2 (100 ng/mL); (4) BMP-9 (100 ng/mL) at 14 days after seeding. (a to h) White-black image views of alizarin red staining on collagen membranes and $(i, j)$ quantified evaluation of alizarin red staining area. $(*$ denotes significant difference, $P<.05)$.

as a 3D scaffold to allow cell ingrowth and repopulation from surrounding tissues. ${ }^{1}$ In addition, collagen serves as an excellent carrier system for growth factors, namely, BMPs. Both PPCM and PDCM behaved similarly by demonstrating approximately $90 \%$ of BMP adsorption onto both PPCM and PDCM and showing slow BMP release over time up to 10 days overall (Fig 2). The sustained delivery of BMPs to bone defects is considered advantageous for long-term bone regeneration compared with a single high-dose burst of BMPs. ${ }^{31,32}$ Therefore, it may be suggested from the present study that both PPCM and PDCM may serve as suitable carrier systems for either rhBMP-2 or rhBMP-9. Furthermore, interestingly, PPCM demonstrated significantly more adsorption and retention of BMP-2 over time, and PDCM demonstrated more retention of BMP-9 from 1 hour to 10 days compared with the other collagen membrane (Fig 2). This difference of kinetics on two collagen membranes might result from the variances of protein 3D structures and binding sites between rhBMP-2 and rhBMP-9.

Thereafter, the cell behavior on collagen membranes combined with rhBMP-2 and rhBMP-9 was investigated on ST2 cell attachment, proliferation, and differentiation (Figs 3 to 6). PPCM demonstrated less cell adhesion compared with the control at 8 hours after cell seeding. Interestingly, however, PDCM showed better cell attachment and comparable results to the control (Fig 3). One potential explanation for this may be because PDCM membranes are denser by nature, 
thereby providing a more easily attachable surface for incoming osteoblasts. It was also found that the additional use of rhBMP-2/rhBMP-9 did not influence cell proliferation on either collagen membrane (Figs $3 \mathrm{c}$ and 3d). Treatment by both collagen membranes showed fewer cell numbers compared with the control; however, cell growth on both collagen membranes was constantly observed for 5 days, and additional rhBMP-2/ rhBMP-9 coating did not influence cell adhesion and proliferation, which suggested relatively biocompatible effects of PPCM and PDCM combined with rhBMP-2 or rhBMP-9. The authors' previous findings have also shown that rhBMP-9 has little effect on cell proliferation and mainly induces rapid differentiation toward osteoblasts. 24,25

Thereafter, osteogenic differentiation was investigated. Although the combination of rhBMP-2 on PDCM increased alizarin red staining (Fig 6j), rhBMP-2 on PPCM did not seem to promote marked increases in osteoblast differentiation. Most interestingly, rhBMP-9 treated samples, regardless of whether they were coated on PPCM or PDCM, demonstrated more potent osteogenic cell differentiation by demonstrating significant increases in ALP and OCN mRNA levels at either 3 or 14 days, ALP activity at 7 days, and alizarin red staining at 14 days (Figs 4 to 6 ). These results are consistent with previous reports by the present authors that rhBMP-9 had the greatest influence on osteoblast differentiation in vitro. ${ }^{24,25}$ Interestingly, while PPCM seemed to increase cell attachment by providing a smoother biomaterial surface, PDCM seemed to favor osteoblast differentiation by providing a more roughened surface. It remains to be further investigated what the molecular mechanism is for these findings and to determine by which signaling pathways rhBMP-9 is able to induce more rapid cellular osteoblast differentiation on the various membranes found in this study. Furthermore, there remains to date no in vivo data comparing the regenerative potential of rhBMP-2 to rhBMP-9 for the regeneration of animal bone defects. Therefore, future animal study is also of vast importance to further characterize the regenerative potential of rhBMP-9 in comparison to the current gold standard rhBMP-2.

\section{CONCLUSIONS}

The results from this study demonstrate that both types of porcine-derived collagen membranes behaved as suitable carriers of rhBMP-2 and rhBMP-9, and both collagen membranes combined with rhBMP-9 demonstrated significantly superior osteopromotive properties compared with the control samples. Interestingly, it was further found that slight differences were observed between two kinds of collagen membranes, including the fact that PDCM membranes seemed to improve cell attachment and proliferation and also seemed to promote greater ALP activity of both rhBMP-2 and rhBMP-9, whereas PPCM membranes showed greater ALP activity when combined with rhBMP-9 only. Nevertheless, these studies point to the necessity to further evaluate both membranes and growth factors in various animal models to further characterize the regenerative potential of both rhBMP-2/ rhBMP-9 in vivo for bone augmentation procedures.

\section{ACKNOWLEDGMENTS}

The authors thank the Department of Cranio-Maxillofacial Surgery, Inselspital at the University of Bern, Switzerland (Chair Professor Tateyuki lizuka) for the financial support of this study. The authors reported no conflicts of interest related to this study.

\section{REFERENCES}

1. Bunyaratavej P, Wang HL. Collagen membranes: A review. J Periodontol 2001;72:215-229.

2. Vignoletti F, Nunez J, Sanz M. Soft tissue wound healing at teeth, dental implants and the edentulous ridge when using barrier membranes, growth and differentiation factors and soft tissue substitutes. J Clin Periodontol 2014;41(suppl):s23-s35.

3. Bottino MC, Thomas V, Schmidt G, et al. Recent advances in the development of GTR/GBR membranes for periodontal regeneration-A materials perspective. Dent Mater 2012;28:703-721.

4. Yukna C, Yukna R. Multi-center evaluation of bioabsorbable collagen membrane for guided tissue regeneration in human Class II furcations. J Periodontol 1996;67:650-657.

5. Lu HK, Lee SY, Lin FP. Elastic modulus, permeation time and swelling ratio of a new porcine dermal collagen membrane. J Periodontal Res 1998;33:243-248.

6. Patino MG, Neiders ME, Andreana S, Noble B, Cohen RE. Collagen as an implantable material in medicine and dentistry. J Oral Implantol 2002;28:220-225.

7. Pabst AM, Happe A, Callaway A, et al. In vitro and in vivo characterization of porcine acellular dermal matrix for gingival augmentation procedures. J Periodontal Res 2014;49:371-381.

8. Shirakata Y, Sculean A, Shinohara Y, et al. Healing of localized gingiva recessions treated with a coronally advanced flap alone or combined with an enamel matrix derivative and a porcine acellular dermal matrix: A preclinical study. Clin Oral Investig 2016;20:1791-1800.

9. Rothamel D, Benner M, Fienitz T, et al. Biodegradation pattern and tissue integration of native and cross-linked porcine collagen soft tissue augmentation matrices - An experimental study in the rat. Head Face Med 2014;10:10.

10. Rothamel D, Schwarz F, Sager M, Herten M, Sculean A, Becker J. Biodegradation of differently cross-linked collagen membranes: An experimental study in the rat. Clin Oral Implants Res 2005;16:369-378.

11. Miron RJ, Zhang YF. Osteoinduction: A review of old concepts with new standards. J Dent Res 2012;91:736-744.

12. King GN, King N, Hughes FJ. Effect of two delivery systems for recombinant human bone morphogenetic protein-2 on periodonta regeneration in vivo. J Periodontal Res 1998;33:226-236.

13. Huang $\mathrm{YH}$, Polimeni G, Qahash M, Wikesjö UM. Bone morphogenetic proteins and osseointegration: Current knowledge - Future possibilities. Periodontol 2000 2008;47:206-223.

14. Jung RE, Glauser R, Schärer $P$, Hämmerle $C H$, Sailer HF, Weber FE. Effect of rhBMP-2 on guided bone regeneration in humans. Clin Oral Implants Res 2003;14:556-568. 
15. Murata M, Maki F, Sato D, Shibata T, Arisue M. Bone augmentation by onlay implant using recombinant human BMP-2 and collagen on adult rat skull without periosteum. Clin Oral Implants Res 2000;11:289-295.

16. Jo JY, Jeong SI, Shin YM, et al. Sequential delivery of BMP-2 and BMP-7 for bone regeneration using a heparinized collagen membrane. Int J Oral Maxillofac Surg 2015;44:921-928.

17. Jung RE, Windisch SI, Eggenschwiler AM, Thoma DS, Weber FE, Hämmerle $\mathrm{CH}$. A randomized-controlled clinical trial evaluating clinical and radiological outcomes after 3 and 5 years of dental implants placed in bone regenerated by means of GBR techniques with or without the addition of BMP-2. Clin Oral Implants Res 2009;20:660-666.

18. Miron RJ, Saulacic N, Buser D, lizuka T, Sculean A. Osteoblast proliferation and differentiation on a barrier membrane in combination with BMP2 and TGF 31 . Clin Oral Investig 2013;17:981-988.

19. Zhang Y, Yang S, Zhou W, Fu H, Qian L, Miron RJ. Addition of a synthetically fabricated osteoinductive biphasic calcium phosphate bone graft to BMP2 improves new bone formation. Clin Implant Dent Relat Res 2016;18:1238-1247.

20. Cheng $\mathrm{H}$, Jiang W, Phillips FM, et al. Osteogenic activity of the fourteen types of human bone morphogenetic proteins (BMPs). J Bone Joint Surg Am 2003;85:1544-1552.

21. Kang $Q$, Sun $M H$, Cheng $H$, et al. Characterization of the distinct orthotopic bone-forming activity of 14 BMPs using recombinant adenovirus-mediated gene delivery. Gene Ther 2004;11:1312-1320.

22. Leblanc E, Trensz F, Haroun S, et al. BMP-9-induced muscle heterotopic ossification requires changes to the skeletal muscle microenvironment. J Bone Miner Res 2011;26:1166-1177.

23. Lamplot JD, Qin J, Nan G, et al. BMP9 signaling in stem cell differentiation and osteogenesis. Am J Stem Cells 2013;2:1-21.
24. Fujioka-Kobayashi M, Sawada K, Kobayashi E, Schaller B, Zhang Y, Miron RJ. Recombinant human bone morphogenetic protein 9 (rhBMP9) induced osteoblastic behaviour on a collagen membrane compared with rhBMP2. J Periodontol 2016;87:e101-e107.

25. Fujioka-Kobayashi M, Sawada K, Kobayashi E, Schaller B, Zhang $Y$, Miron RJ. Osteogenic potential of rhBMP9 combined with a bovine-derived natural bone mineral scaffold compared to rhBMP2. Clin Oral Implants Res 2017;28:381-387.

26. Miron RJ, Bosshardt DD, Gemperli AC, et al. In vitro characterization of a synthetic calcium phosphate bone graft on periodontal ligament cell and osteoblast behavior and its combination with an enamel matrix derivative. Clin Oral Investig 2014;18:443-451.

27. Miron RJ, Bosshardt DD, Buser D, et al. Comparison of the capacity of enamel matrix derivative gel and enamel matrix derivative in liquid formulation to adsorb to bone grafting materials. J Periodontol 2015;86:578-587.

28. Fujioka-Kobayashi M, Sawada K, Kobayashi E, Schaller B, Zhang Y, Miron RJ. Osteogenic potential of rhBMP9 combined with a bovine-derived natural bone mineral scaffold compared to rhBMP2. Clin Oral Implants Res 2017;28:381-387.

29. Miron RJ, Hedbom E, Saulacic N, et al. Osteogenic potential of autogenous bone grafts harvested with four different surgical techniques. J Dent Res 2011;90:1428-1433.

30. Hämmerle $\mathrm{CH}$, Jung $\mathrm{RE}$, Feloutzis $\mathrm{A}$. A systematic review of the survival of implants in bone sites augmented with barrier membranes (guided bone regeneration) in partially edentulous patients. J Clin Periodontol 2002;29(suppl):s226-s231.

31. Woo BH, Fink BF, Page R, et al. Enhancement of bone growth by sustained delivery of recombinant human bone morphogenetic protein-2 in a polymeric matrix. Pharm Res 2001;18:1747-1753.

32. Hollinger JO, Uludag $\mathrm{H}$, Winn SR. Sustained release emphasizing recombinant human bone morphogenetic protein-2. Adv Drug Deliv Rev 1998;31:303-318. 